\title{
Sudden Cardiac Death Survival Prediction from Restitution Dispersion Analysis
}

\author{
Julia Ramírez ${ }^{1,2}$, Ana Mincholé ${ }^{3}$, Juan Bolea ${ }^{1,2}$, Pablo Laguna ${ }^{1,2}$, Esther Pueyo ${ }^{2,1}$ \\ ${ }^{1}$ GTC (Grupo de Tecnologías de las, Comunicaciones)
Instituto de Investigacion en Ingeniería de Aragon (I3A). \\ Universidad de Zaragoza, Mariano Esquillor s/n, 50018, Zaragoza, Spain. \\ Tel. +34-976762707, Fax +34-976762043, e-mail: Julia.Ramirez@unizar.es \\ ${ }^{2}$ CIBER - Bioingeniería, Biomateriales y Nanomedicina, Spain \\ ${ }^{3}$ University of Oxford, Oxford, United Kingdom
}

\begin{abstract}
Increase in the dispersion of action potential duration restitution (APDR) has been associated with sudden cardiac death (SCD). A marker, $\Delta \alpha$, was proposed to quantify APDR dispersion from the electrocardiogram (ECG). 609 ECG recordings were analysed. The marker $\Delta \alpha$ stratified patients according to their risk of suffering from SCD.
\end{abstract}

\section{Introduction}

APDR measures the relationship between the action potential duration and the RR interval at steady-state pacing. Due to heterogeneities in the ventricles, APDR presents spatial variations generally termed APDR dispersion. An increase in APDR dispersion has been associated with higher propensity to suffer from ventricular arrhythmias and SCD. Recently, a marker, $\Delta \alpha$, which accounts for the rate normalized differences of the $T_{\text {pe }}$ interval, was proposed to quantify APDR dispersion from the ECG at steady-state conditions [1].

\section{Materials and methods}

\section{Materials}

Consecutive patients were enrolled in the MUSIC (MUerte Súbita en Insuficiencia Cardiaca) study, a prospective, multicenter study designed to assess risk predictors for cardiovascular mortality in ambulatory patients. The Holter recordings of 609 patients (48 victims of SCD, 64 of other cardiac causes, 25 of non-cardiac death causes and 472 survivors) with sinus rhythm were available for the present study. Each recording consisted of 3 ortogonal ECG leads, sampled at $200 \mathrm{~Hz}$. In this study, the population in the database was splitted into two groups: SCD victims (group 1) and victims of other cardiac causes, non-cardiac causes and survivors (group 2).
Patients were followed up every 6 months for a median of 48 months. $S C D$ was defined as (1) a witnessed death occurring within 60 minutes from the onset of new symptoms unless a cause other than cardiac failure was obvious, (2) an unwitnessed death ( $<24$ hours) in the absence of preexisting progressive circulatory failure or other causes of death, or (3) death during attempted resuscitation.

\section{Methods}

Preprocessing of the ECG signals included low pass filtering at $40 \mathrm{~Hz}$ to remove electric and muscle noise, cubic splines interpolation for baseline wander removal and ectopic beats detection.

Principal Component Analysis was applied over the three leads to emphasize T-wave energy and improve delineation. A Single-Lead-and-rules delineation technique was applied to mark the onsets and offsets of the T-wave. From the annotation marks, RR, QT and Tpe series were obtained and subsequently interpolated at a sampling frequency of $1 \mathrm{~Hz}$.

The spatial dispersion of APDR slopes was estimated from the ECG as

$$
\Delta \alpha=\frac{\partial T p e}{\partial R R}
$$

measured at steady-state RR intervals [1].

\section{Results and discussion}

The mean value of $\Delta \alpha$ in the study population was $0.028 \pm 0.076$ and the 25th, 50th and 75th percentiles were $0.005,0.022$ and 0.046 , respectively. 
$\Delta \alpha$ discriminated between the group formed by SCD victims (group 1 ) and the group composed of the other patients (group 2), with mean \pm SEM values of: $\Delta \alpha=0.052 \pm 0.013$ for the former and $\Delta \alpha$ $=0.026 \pm 0.003$ for the latter $(p=.048)$. Patients were divided into $\Delta \alpha$ positive $(\Delta \alpha+)$ and negative $(\Delta \alpha-)$ groups by setting a cut-off point of 0.046 for $\Delta \alpha$, corresponding to the 75th percentile of the distribution of $\Delta \alpha$ in the population. Of the 609 patients studied, 457 (75.0\%) were included in the $\Delta \alpha$ - group $(\Delta \alpha \leq 0.046)$ and $152(25 \%)$ in the $\Delta \alpha+$ group $(\Delta \alpha>0.046)$. A two-tailed Fisher exact test showed an existing effect of being a SCD victim on having $\Delta \alpha>0.046(\mathrm{p}=.003)$, with a survival rate higher in the $\Delta \alpha$ - group for SCD endpoint. In a survival analysis, Cox regression revealed that a $\Delta \alpha+$ outcome was associated with SCD $(p=.001)$, as shown in Figure 1.

\section{Conclusions}

This study demonstrates that quantification of APDR from the ECG is a strong predictor of SCD. This finding supports the hypothesis that elevated APDR dispersion reflects abnormal cardiac function predisposing to SCD.

\section{REFERENCIAS}

[1] Mincholé, A., Pueyo, E., Rodríguez, J.F., Zacur, E., Doblaré, M. and Laguna, P. Quantification of restitution dispersion from the dynamic changes of the T-wave peak to end, measured at the surface ECG. IEEE Transactions on Biomedical Engineering, vol. 58, 2011, pp. 1172-1182.
[2] Pueyo, E., Smetana, P., Caminal, P., de Luna, A.B., Malik, M. and Laguna, P. Characterization of QT interval asaptation to $\mathrm{RR}$ interval changes and its use as a risk-stratifier of arrhythmic mortality in amiodarone-treated survivors of acute myocardial infarction. IEEE Transactions on Biomedical ENgineering, vol. 51, 2004, pp. 1511-1520.

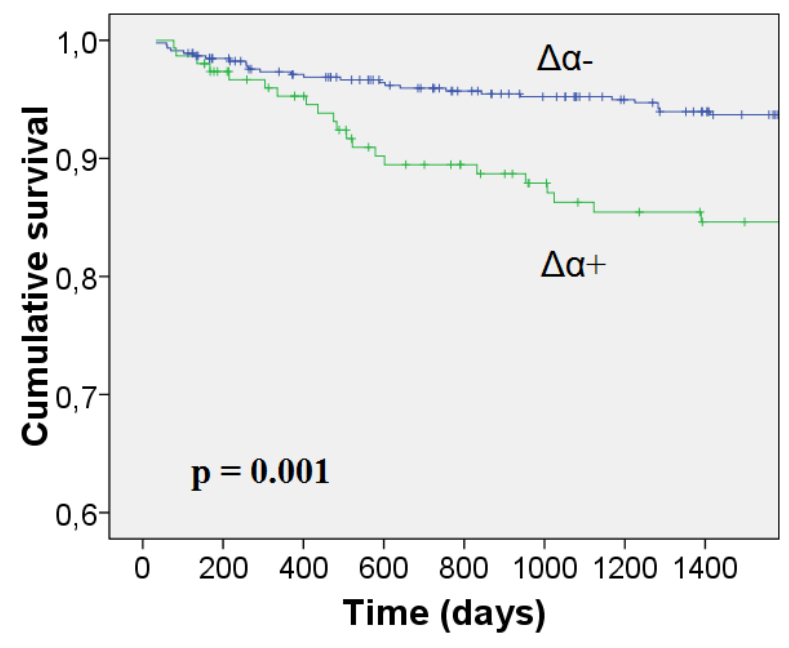

Fig 1: Event-free curves for SCD 